\title{
Malaria Screener: a smartphone application for automated malaria screening
}

\author{
Hang Yu', Feng Yang ${ }^{1}$, Sivaramakrishnan Rajaraman', Ilker Ersoy², Golnaz Moallem ${ }^{1,3}$, Mahdieh Poostchi', \\ Kannappan Palaniappan ${ }^{4}$, Sameer Antani ${ }^{1}$, Richard J. Maude ${ }^{5,6,7}$ and Stefan Jaeger ${ }^{1 *}$ (D)
}

\begin{abstract}
Background: Light microscopy is often used for malaria diagnosis in the field. However, it is time-consuming and quality of the results depends heavily on the skill of microscopists. Automating malaria light microscopy is a promising solution, but it still remains a challenge and an active area of research. Current tools are often expensive and involve sophisticated hardware components, which makes it hard to deploy them in resource-limited areas.

Results: We designed an Android mobile application called Malaria Screener, which makes smartphones an affordable yet effective solution for automated malaria light microscopy. The mobile app utilizes high-resolution cameras and computing power of modern smartphones to screen both thin and thick blood smear images for $P$. falciparum parasites. Malaria Screener combines image acquisition, smear image analysis, and result visualization in its slide screening process, and is equipped with a database to provide easy access to the acquired data.

Conclusion: Malaria Screener makes the screening process faster, more consistent, and less dependent on human expertise. The app is modular, allowing other research groups to integrate their methods and models for image processing and machine learning, while acquiring and analyzing their data.
\end{abstract}

Keywords: Automated light microscopy, Smartphone application, Malaria, Machine learning, Convolutional neural network

\section{Background}

Microscopic examination of stained blood smears is still considered the gold standard for malaria diagnosis $[1,2]$. It offers the ability to characterize parasite species, quantify parasite density, and assess the effectiveness of antimalarial treatment. However, regions that are suffering from the disease are often lacking in well-trained personnel that can perform high-quality microscopy examination due to the high costs to train such experts $[3,4]$. Besides, the examination process can be very time-consuming and error-prone.

To address these issues, there have been attempts to automate both image acquisition and image analysis for

\footnotetext{
* Correspondence: stefan.jaeger@nih.gov

'Lister Hill National Center for Biomedical Communications, National Library of Medicine, National Institutes of Health, Bethesda, MD 20894, USA

Full list of author information is available at the end of the article
}

the microscopic examination of blood smears. Gopakumar, G.P. et al. [5] proposed a custom-built portable slide scanner that automatically collects and analyzes focus stacks of blood smear images. Muthumbi, A. et al. [6] proposed a system that adds a programmable LED array to the standard microscope, and uses a large-fieldof-view, low-resolution objective lens to capture thousands of cells in one snapshot. While these methods show great potential, they are often hard to test on a large scale, especially in resource-limited settings, due to the difficulty to replicate their sophisticated hardware design. Other research work [7-9] concentrates on image analysis algorithms. They tend to be lacking a user interface to put their systems to use in real clinical settings.

In this paper, we present a smartphone-based semiautomated system that provides analysis of blood smear 
images for malaria screening, with an easy-to-use user interface. Our Android smartphone application combines multiple functions, including image acquisition, image screening, and management of the acquired data. The smartphone is used in combination with a microscope adapter as shown in (Fig. 1), which is a very affordable setup by design. Android smartphones and microscopes are commonly available in malaria clinics, and an adapter is usually inexpensive. For example, the universal smartphone microscope adapter we use costs less than $\$ 100$ (from telescopeadapters.com, model: USPA2). The low-cost design and easy-to-use interface give the system great potential to assist malaria diagnosis in resource-limited areas. Furthermore, the modular architecture allows it to be adapted by fellow researchers to advance their study.

\section{Implementation}

\section{Software architecture}

We designed Malaria Screener by following objectoriented principles. A diagram of the application's architecture is shown in Fig. 2. It consists of three independent modules, including: a slide screening module, a data management module, and a data upload module. The slide screening module, being the core of the system, has three sub-modules that work sequentially to perform image acquisition, parasite detection, and result visualization, respectively. The data management module stores the images and the corresponding metadata acquired during slide screening sessions, giving user access to previously screened slides. Finally, the data upload module transfers the local data to an online repository for record-keeping and further training of the system. We implemented the front end user interface (UI) based on Android API while the back-end of the application is powered by a mix of different libraries including OpenCV4Android (opencv.org/android/), TensorFlow Lite [16], SQLite [18], and Box API [19].

Malaria Screener is designed to be easily extendable and customizable. The source code is hosted on GitHub as an open-source project; fellow researchers can modify

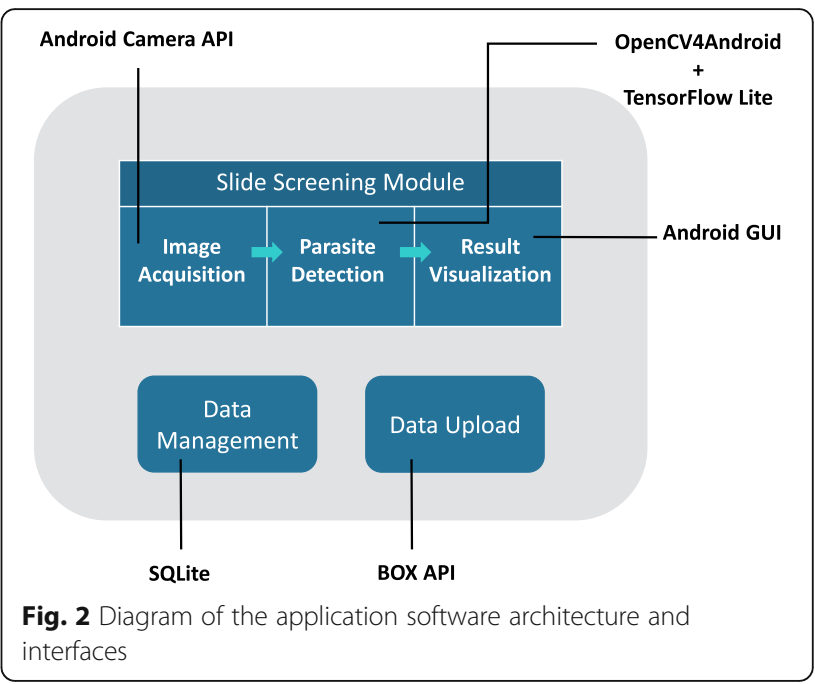

the code to suit their needs. For example, developers can replace the parasite detection module to test their detection algorithm, or can add another classifier to detect other malaria parasite species

\section{Critical components \\ Slide screening module}

As mentioned above, three independent sub-modules work sequentially to screen a slide for malaria parasites. The image acquisition module is the first module in this pipeline. For this module, we implemented a customized camera function using the Android Camera API [10]. This includes a Camera object that controls the intrinsic parameters of the camera hardware, and a CameraPreview object that displays the preview image to the user. During a screening session, the user presses a button to capture the image when a suitable field of view becomes visible. To obtain the best image quality possible, the Camera object requests the maximum resolution that the smartphone camera offers and saves the captured image as PNG, a lossless compression format.

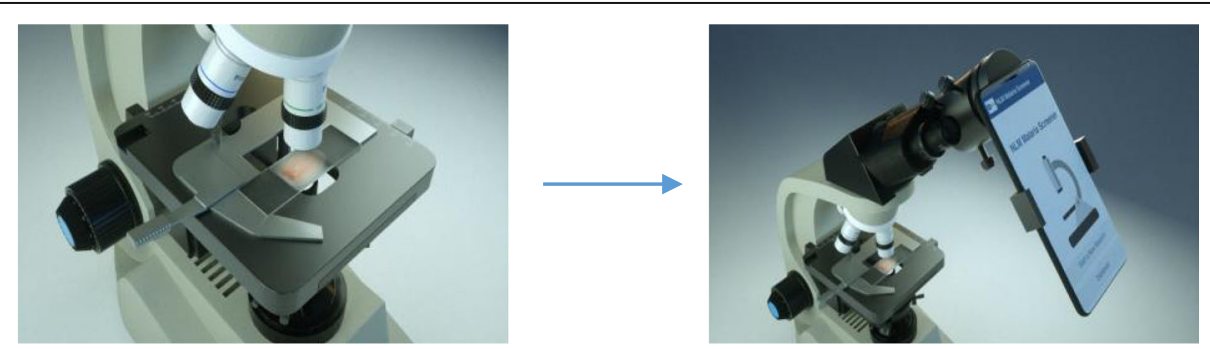

Fig. 1 System Setup for Malaria Screener. During the (semi-) automated* screening process, the body of the smartphone is attached to an adapter. The adapter holds the phone, and aligns its camera with the eyepiece of the microscope. * The system is semi-automated in that the user needs to move the slide manually to search for an ideal field of view while capturing smear images 
The captured image is then passed to the parasite detection module as input. Malaria Screener can examine both thin and thick smears with potential $P$. falciparum infections. The performance evaluation of the detection module can be found in previous publications [11-14]. Figure 3 illustrates how an image is processed in this module. For a single thin smear image, the goal is to detect the number of infected red blood cells (RBCs) and the total number of RBCs in the image. For a thick smear image, on the other hand, the goal is to detect the number of parasites and white blood cells (WBCs). The parasite detection module has a ThinSmearProcessor class and a ThickSmearProcessor class to handle each of the two scenarios. With ThinSmearProcessor, a thin smear image is first segmented to detect RBCs. Small cell patches of $\mathrm{RBCs}$ are cropped from the original image. With ThickSmearProcessor, parasite candidate patches that cover the typical size of a parasite are cropped from a thick smear image. Both classes use pre-trained Convolutional Neural Network (CNN) models to make binary classifications: infected vs uninfected $R B C$ in the case of a thin smear, or parasite vs background in the case of a thick smear. The CNN models are pretrained on a PC with TensorFlow and Keras, which outputs the trained models in HDF5 (.h5) format. Next, the models are converted to Protocol Buffers (.pb) format [15] and deployed to the app using TensorFlow Lite [16, 17].

The result visualization module uses ResultDisplayerActivity, a UI class that was implemented to present the detection results to the user (Fig. 5 (a)(3)). This class generates a down-sampled version of the captured image with labels drawn on the infected RBCs (parasites for thick smear images). In addition, it uses a table to show the numerical results. Together, these two outputs visualize the computational result of the input smear for the user.

\section{Data management module}

The app stores images and corresponding metadata locally on the phone. Images are stored in a designated folder of the internal storage. Within this folder, images from the same screening session are grouped in their own subfolders. Metadata of the images is stored in a local SQLite database [18]. The database includes four tables: a patient table, a slide table, a thin smear image table, and a thick smear image table. Figure 4 shows the structure of the database in more detail. The data management module also offers a UI to let the user browse the images and metadata stored in the SQLite database, as shown in (Fig. 5 (a)(5)).

\section{Data upload module}

Images and metadata in the database can be exported and uploaded to an online repository. The uploaded data can be used to examine the app performance, and to improve the classifier of the parasite detection module with additional training.

An upload event can be initiated in two different ways. The first option is to start an upload event from the database UI. With this option, the app will scan for all data that has not been uploaded yet, which will then be uploaded. However, this type of bulk upload can be a very heavy task, which can take a long time since there might be several gigabytes of images to be uploaded. Therefore, we implemented another upload option in which the app attempts to trigger an upload event after each screening session. As long as a Wi-Fi connection can be detected, this event will try to upload the data from the current session.

The back-end of this module is implemented with a mixture of both Android and Box API [19]. Android Service class and Thread class $[20,21]$ are used to implement the upload function which executes the upload tasks in a separate thread. It allows the user to continue with other things while the upload tasks proceed in the

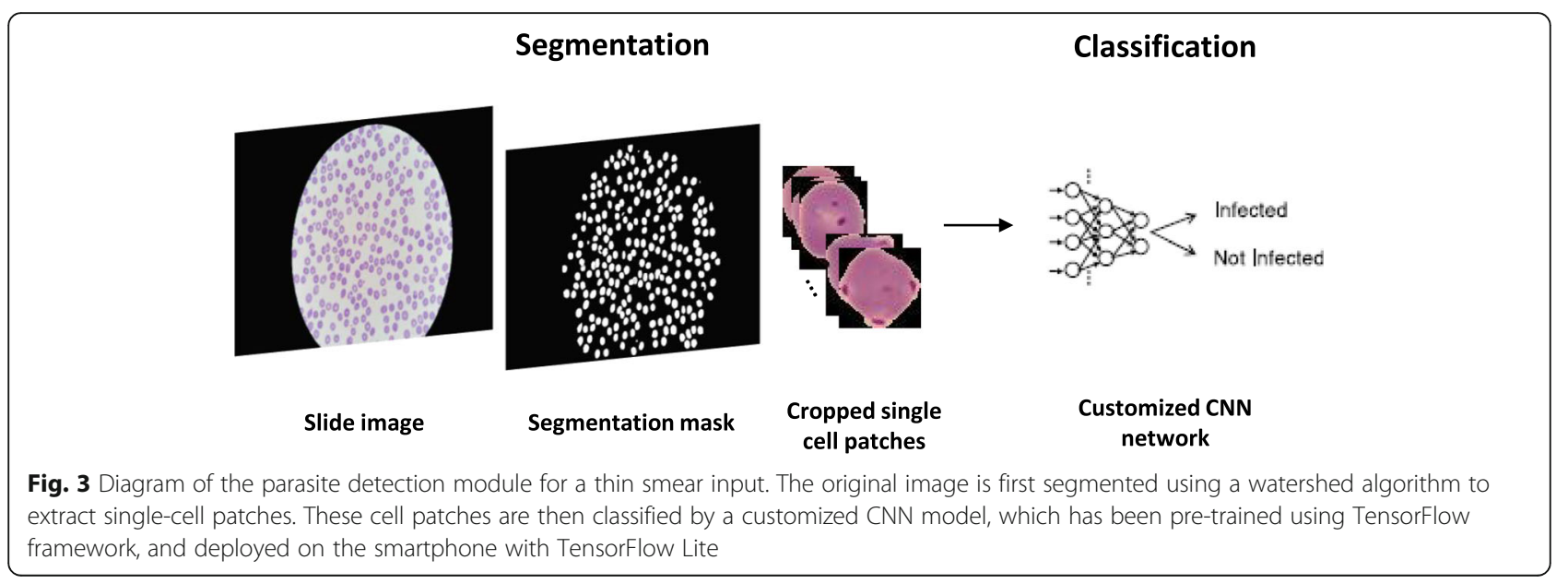




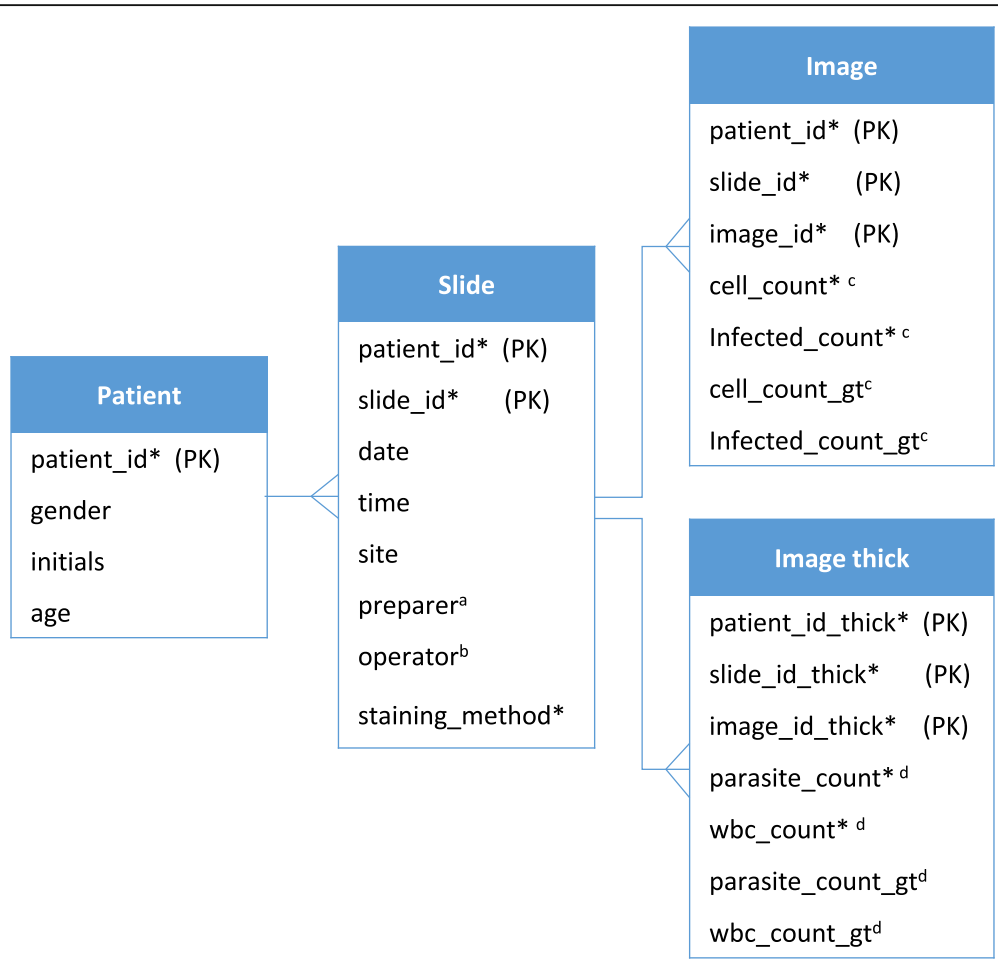

Fig. 4 Diagram of the local SQLite database. PK: primary key. Each line that connects two tables indicates the one-to-many relationship between them. For example, the patient table has a one-to-many relationship with the Slide table, meaning one patient can have multiple slides. Fields with an asterisk symbol $\left(^{*}\right)$ are either mandatory inputs by the user or automatically generated data; other fields are optional inputs by the user. ${ }^{a}$ Name of the slide preparer. ${ }^{b}$ Name of the user performing the screening. ${ }^{C}$ App outputs and manual counts for thin smears: RBC counts, infected RBC counts, manual RBC counts, manual infected RBC counts. ${ }^{d}$ App outputs and manual counts for thick smears: parasite counts, WBC counts, manual parasite counts, manual WBC counts

background. Box API is used to implement functions to execute upload tasks to a Box repository.

\section{Results}

A fast and effective mobile app is developed as a lightweight solution to automated malaria light microscopy. This section describes its workflow during a slide screening session. Due to space constraints, this section only illustrates the important parts of the screening pipeline. For more details, readers should refer to the user manual, which can be downloaded together with the source code, see download link in Availability of Data and Materials. The workflow proceeds in six steps as follows, with each step corresponding to a panel in (Fig. 5 (a)):

(1) Once the smartphone is setup on top of the microscope, using an adapter, the user can start a session from the main page of the app.

(2) A preview screen of the camera is presented to the user at the beginning of a session, and the user can use the button at the top of the screen to set the smear type (thin or thick) at this point. Then, the user can search for a suitable field of view on the slide, and press the camera button to capture the image.

(3) The app will then start to process the captured image, and will display a result visualization page on the screen where it shows the detection results. For example, in thin smear mode, the number of infected and total RBCs are shown as well as a running total. The app also shows a result image with the infected RBCs marked in red. Step (2) and (3) are repeated (Fig. 5 (b)) while the user captures more smear images. The iteration stops when the total number of RBCs reaches a user-determined maximum.

(4) Next, the app goes through several screens to let the user enter relevant information about the slide, such as slide ID, staining method, and hematocrit value.

(5) Then, the session ends. Both the images and metadata are saved locally, and can be viewed through the database UI at a later time.

(6) Finally, the app triggers an upload event to send the saved data to the central Box repository. Meanwhile, a floating widget hovers over the app screen to show the upload progress. 
a

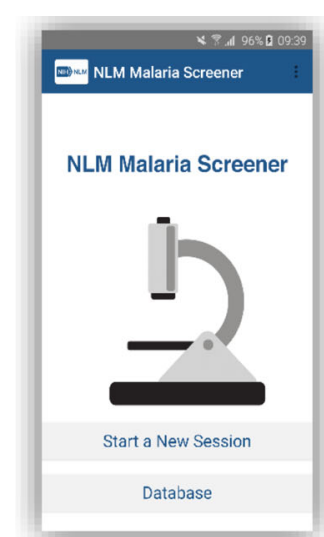

(1) Start screen

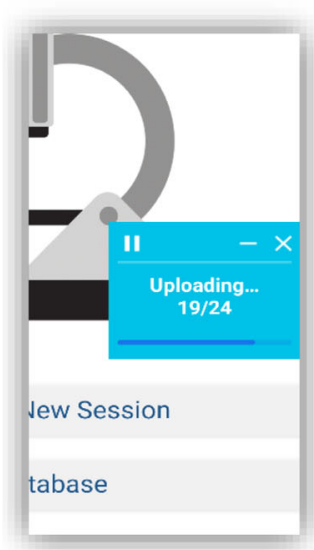

(6) Data upload

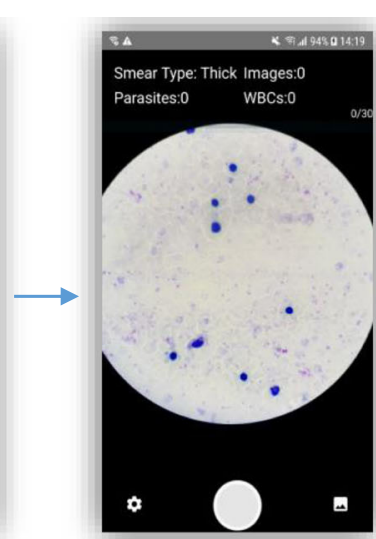

(2) Image acquisition

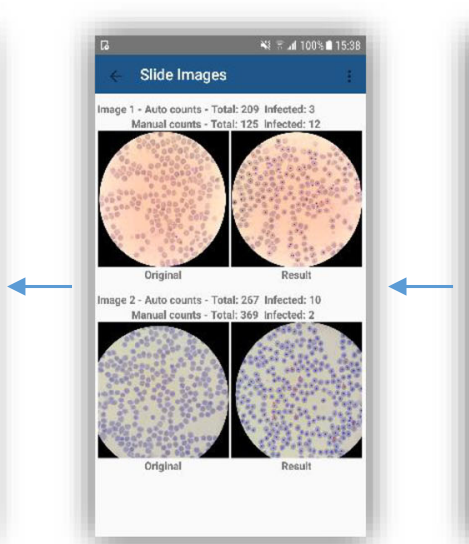

(5) Database

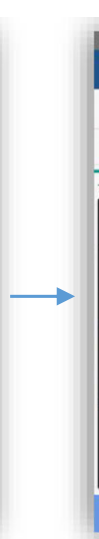

(3) Result visualization

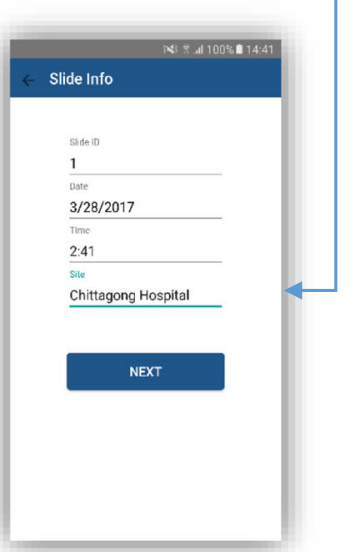

(4) Slide info entry

b

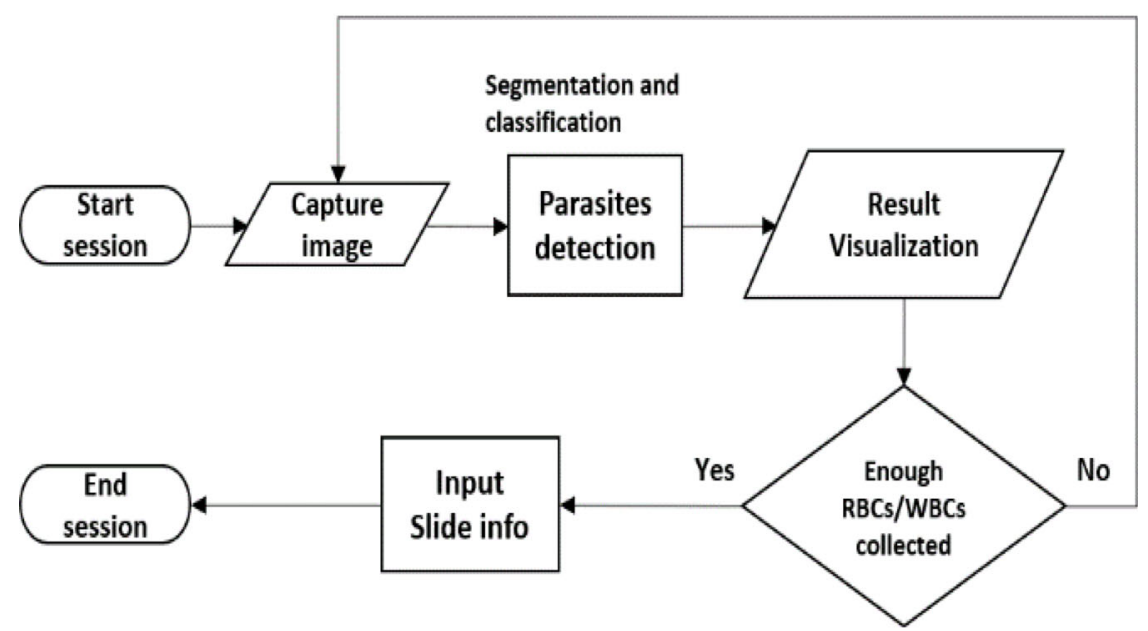

Fig. 5 a UI screens during a slide screening session. $\mathbf{b}$ The workflow of a session 
Table 1 System mean performance on five folds for thick smears

\begin{tabular}{llllll}
\hline & Accuracy & AUC & Sensitivity & Specificity & Precision \\
\hline $\begin{array}{l}\text { Patch-level } \\
\text { [12] }\end{array}$ & $96.89 \%$ & $98.48 \%$ & $90.82 \%$ & $97.43 \%$ & $74.84 \%$ \\
$\begin{array}{l}\text { Patient-level } \\
\text { [12] }\end{array}$ & $78.00 \%$ & $84.90 \%$ & $79.33 \%$ & $74.00 \%$ & $90.42 \%$ \\
\hline
\end{tabular}

\section{Testing}

Tests were performed with the algorithms we implemented for the slide screening module. We acquired and annotated Giemsa-stained thick and thin blood smear images from 150 patients infected with P. falciparum, and from 50 normal patients, at Chittagong Medical College Hospital, Bangladesh.

For thick smear, we evaluated the performance of our system with five-fold cross validation, using 2967 thick blood smear images from these 200 patients: 1819 images from 150 infected patients and 1148 images from 50 normal patients. Table 1 shows the mean performance of our system on five folds at both patch and patient level [12].

For thin smear, we also performed five-fold cross validation at both patch and patient level [14]. We compared our results with the state-of-the-art on patch level, as shown in Table 2. To the best of our knowledge, we could find no comparable literature that performed cross-validation studies on patient-level.

More details can be found in our previous publications [11-14, 27]. We are currently in the process of field testing our app with collaborators around the world.

\section{Discussion}

Malaria Screener is a step towards automating malaria light microscopy. It provides a solution to improve malaria point-of-care diagnosis in the field. To the best of our knowledge, Malaria Screener is the first smartphone-based system that can screen thin and thick smears. In addition to the basic slide screening functions, which are based on computational image analysis and machine learning, we try to integrate additional functions into our mobile app to support the daily work of malaria field workers. In particular, the data management function can be very helpful. Users can enter patient information directly into the app's database, thus avoiding the trouble of using a separate system to manage the data.

For malaria research, the app offers a powerful and efficient tool for field tests and data collection, which are usually done through a collaboration between medical imaging research groups and hospitals. Coordinating the protocols typically requires a considerable effort, involving data processing and formatting. Malaria Screener solves this problem by integrating a slide screening module, a database module, and a data upload module into the same smartphone application, making slide screening and data collection a streamlined process that generates and delivers ready-to-use data.

Finally, with the release of the current codebase of the software as an open-source project, we anticipate it to serve groups that are new to this field of research. The modular design allows other developers to build upon the current implementation. For example, our parasite detection algorithm can be easily swapped, allowing other groups to test their own algorithms. By making Malaria Screener an open-source project, we are hoping to provide a platform for the scientific community to work together and to advance the automation of malaria diagnosis.

\section{Conclusions}

We present a fast, low-cost smartphone application for malaria screening. We demonstrate that the app offers important functionalities with an intuitive user interface to (a) screen slides and count infected red blood cells and parasites in thin and thick smear images automatically for $P$. falciparum malaria, and (b) to manage the images and metadata generated throughout the screening process, which can be used to further optimize the image analysis model.

Table 2 Classification module mean performance on five folds for thin smears compared to the state-of-the-art

\begin{tabular}{|c|c|c|c|c|c|}
\hline & Accuracy & AUC & Sensitivity & Specificity & F1-score \\
\hline Proposed Module (Patch-level) [14] & $98.6 \%$ & $99.9 \%$ & $98.1 \%$ & $99.2 \%$ & $98.7 \%$ \\
\hline Proposed Module (Patient-level) [14] & $95.9 \%$ & $99.1 \%$ & $94.7 \%$ & $97.2 \%$ & $95.9 \%$ \\
\hline Gopakumar et al. (2018) [5] & $97.7 \%$ & - & $97.1 \%$ & $98.5 \%$ & - \\
\hline Bibin, Nair \& Punitha (2017) [22] & $96.3 \%$ & - & $97.6 \%$ & $95.9 \%$ & - \\
\hline Dong et al. (2017) [23] & $98.1 \%$ & - & - & - & - \\
\hline Liang et al. (2017) [24] & $97.3 \%$ & - & $96.9 \%$ & $97.7 \%$ & - \\
\hline Das et al. (2013) [25] & $84.0 \%$ & - & $98.1 \%$ & $68.9 \%$ & - \\
\hline Ross et al. (2006) [26] & $73.0 \%$ & - & $85.0 \%$ & - & - \\
\hline
\end{tabular}


Based on the promising results from previous tests, and interest shown by the research community, we anticipate this project to serve as a code base for future developments in this area.

\section{Availability and requirements \\ Project name: Malaria Screener. \\ Project home page: https://lhncbc.nlm.nih.gov/project/ malaria-screener \\ Operating System: Android. \\ Programming language: Java, $\mathrm{C}++$ (for Android Native development). \\ Other requirements: Android Lollipop/5.0 and above. \\ License: Open Source Software. \\ Any restrictions to use by non-academics: N/A. \\ Abbreviations \\ GUI: Graphical user interface; API: Application programming interface; RBC: Red blood cell; WBC: White blood cell; CNN: Convolutional neural network; PK: Primary key}

\section{Acknowledgments}

We would like to thank Dr. Kamolrat Silamut and Urmila Sampathkumar for helping with the data acquisition and annotation.

\section{Authors' contributions}

HY wrote the mobile app and prepared the manuscript. FY developed the algorithm for thick smear processing. SR provided the CNN model for thin smear classification. IE provided the segmentation algorithm for thin smear segmentation. GM provided the algorithm for WBC segmentation. MP contributed to the algorithm for thin smear processing. KP provided the too for labeling ground truth data. SA managed the project. RM provided guidance to the project with his medical expertise, and organized data acquisition. SJ managed the project and edited the manuscript. All authors read and approved the final manuscript.

\section{Funding}

This research is supported by the Intramural Research Program of the National Institutes of Health (NIH), National Library of Medicine (NLM), and Lister Hill National Center for Biomedical Communications (LHNCBC). Mahidol-Oxford Tropical Medicine Research Unit is funded by the Wellcome Trust of Great Britain. This work was partially supported by U.S. NIH National Institute of Neurological Disorders and Stroke award R01NS110915 (KP).

\section{Availability of data and materials}

Source Code is available to download at https://github.com/n/m-malaria/ MalariaScreener.

\section{Ethics approval and consent to participate}

We photographed Giemsa-stained blood smear slides from 150 P. falciparum infected patients and 50 normal patients at Chittagong Medical College Hospital, Bangladesh. We de-identified all images and their annotations, and archived them at the National Library of Medicine (IRB\#12972).

\section{Consent for publication}

Not applicable.

\section{Competing interests}

The authors declare that they have no competing interests.

\section{Author details}

${ }^{1}$ Lister Hill National Center for Biomedical Communications, National Library of Medicine, National Institutes of Health, Bethesda, MD 20894, USA. ${ }^{2}$ Institute for Data Science and Informatics, University of Missouri-Columbia, Columbia, MO 65211, USA. ${ }^{3}$ Electrical and Computer Engineering Department, Texas Tech University, Lubbock, TX 79409, USA. ${ }^{4}$ Electrical Engineering and Computer Science Department, University of
Missouri-Columbia, Columbia, MO 65211, USA. ${ }^{5}$ Mahidol Oxford Tropical Medicine Research Unit, Mahidol University, Bangkok 10400, Thailand. ${ }^{6}$ Centre for Tropical Medicine and Global Health, Nuffield Department of Medicine, University of Oxford, Oxford, UK. ${ }^{7}$ Harvard TH Chan School of Public Health, Harvard University, Boston, USA.

Received: 20 May 2020 Accepted: 24 September 2020

Published online: 11 November 2020

\section{References}

1. World Health Organization: World Malaria Report, 2018. (2018).

2. WHO: Guidelines for the Treatment of Malaria, Third edition. World Health Organization (2015).

3. Kiggundu M, Nsobya SL, Kamya MR, Filler S, Nasr S, Dorsey G, Yeka A. Evaluation of a comprehensive refresher training program in malaria microscopy covering four districts of Uganda. Am J Trop Med Hyg. 2011; 84(5):820-4.

4. WHO: Malaria micropscopy quality assurance manual. World Health Organization (2016).

5. Gopakumar GP, Swetha M, Sai Siva G, Sai Subrahmanyam GR. Convolutional neural network-based malaria diagnosis from focus stack of blood smear images acquired using custom-built slide scanner. J Biophotonics. 2018; 11(3):e201700003.

6. Muthumbi A, Chaware A, Kim K, Zhou KC, Konda PC, Chen R, Judkewitz B, Erdmann A, Kappes B, Horstmeyer R. Learned sensing: jointly optimized microscope hardware for accurate image classification. Biomed Opt Exp. 2019;10(12):6351-69.

7. Poostchi M, Silamut K, Maude RJ, Jaeger S, Thoma G. Image analysis and machine learning for detecting malaria. Transl Res. 2018;194:36-55.

8. Oliveira AD, Prats C, Espasa M, Serrat FZ, Sales CM, Silgado A, Codina DL, Arruda ME, i Prat JG, Albuquerque J. The malaria system microApp: a new, mobile device-based tool for malaria diagnosis. JMIR Res Protocols. 2017; 6(4):e70.

9. Rosado L, Da Costa JM, Elias D, Cardoso JS. Mobile-based analysis of malaria-infected thin blood smears: automated species and life cycle stage determination. Sensors. 2017;17(10):2167.

10. Android Camera API. Android developer guides. Retrieved from https:// developer.android.com/guide/topics/media/camera. Accessed 23 April 2020

11. Yang F, Poostchi M, Yu H, Zhou Z, Silamut K, Yu J, Maude RJ, Jaeger S, Antani S. Deep Learning for Smartphone-based Malaria Parasite Detection in Thick Blood Smears. IEEE journal of biomedical and health informatics. 2019 Sep 23.

12. Yang F, Yu H, Silamut K, Maude RJ, Jaeger S, Antani S. Smartphonesupported malaria diagnosis based on deep learning. International workshop on machine learning in medical imaging 2019 Oct 13 (pp. 73-80). Springer, Cham.

13. Rajaraman S, Jaeger S, Antani SK. Performance evaluation of deep neural ensembles toward malaria parasite detection in thin-blood smear images. PeerJ. 2019;7:e6977

14. Rajaraman S, Antani SK, Poostchi M, Silamut K, Hossain MA, Maude RJ Jaeger S, Thoma GR. Pre-trained convolutional neural networks as feature extractors toward improved malaria parasite detection in thin blood smear images. PeerJ. 2018;6:e4568.

15. Protocol Buffers. Android developer guides. Retrieved from https:// developers.google.com/protocol-buffers/. Accessed 23 April 2020.

16. Get started with TensorFlow Lite. TensorFlow guide. Retrieved from https:// www.tensorflow.org/lite/guide/get_started\#1_choose_a_model. Accessed 23 April 2020

17. Toni, T. (2017, March 29th). Deploying a TensorFlow model to Android. Retrieved from https://medium.com/joytunes/deploying-a-tensorflowmodel-to-android-69d04d1b0cba Last accessed: 4/23/2020.

18. Saving data using SQLite. Android developer guides. Retrieved from https://developer.android.com/training/data-storage/sqlite\#java. Accessed 23 April 2020

19. Box custom app. Box development guides. Retrieved from https:// developer.box.com/guides/applications/. Accessed 23 April 2020.

20. Android Service. Android developer guides. Retrieved from https:// developer.android.com/reference/android/app/Service. Accessed 23 April 2020

21. Thread. Android developer guides. Retrieved from https://developer.android com/reference/java/lang/Thread. Accessed 23 April 2020. 
22. Bibin D, Nair MS, Punitha P. Malaria parasite detection from peripheral blood smear images using deep belief networks. IEEE Access. 2017:5:9099108.

23. Dong $Y$, Jiang $Z$, Shen $H$, Pan WD, Williams LA, Reddy W, Benjamin WH, Bryan AW. Evaluations of deep convolutional neural networks for automatic identification of malaria infected cells. In2017 IEEE EMBS international conference on Biomedical \& Health Informatics (BHI) 2017 Feb 16 (pp. 101104). IEEE.

24. Liang Z, Powell A, Ersoy I, Poostchi M, Silamut K, Palaniappan K, Guo P, Hossain MA, Sameer A, Maude RJ, Huang JX. CNN-based image analysis for malaria diagnosis. In2016 IEEE international conference on bioinformatics and biomedicine (BIBM) 2016 Dec 15 (pp. 493-496). IEEE.

25. Das DK, Ghosh M, Pal M, Maiti AK, Chakraborty C. Machine learning approach for automated screening of malaria parasite using light microscopic images. Micron. 2013;45:97-106.

26. Ross NE, Pritchard CJ, Rubin DM, Duse AG. Automated image processing method for the diagnosis and classification of malaria on thin blood smears. Med Biol Eng Comput. 2006;44(5):427-36.

27. Poostchi M, Ersoy I, McMenamin K, Gordon E, Palaniappan N, Pierce S, Maude RJ, Bansal A, Srinivasan P, Miller L, Palaniappan K, Thoma G, and Jaeger S. Malaria parasite detection and cell counting for human and mouse using thin blood smear microscopy. Journal of Medical Imaging, International Society for Optics and Photonics. 2018;5(4):1-14.

\section{Publisher's Note}

Springer Nature remains neutral with regard to jurisdictional claims in published maps and institutional affiliations.

Ready to submit your research? Choose BMC and benefit from:

- fast, convenient online submission

- thorough peer review by experienced researchers in your field

- rapid publication on acceptance

- support for research data, including large and complex data types

- gold Open Access which fosters wider collaboration and increased citations

- maximum visibility for your research: over $100 \mathrm{M}$ website views per year

At $\mathrm{BMC}$, research is always in progress.

Learn more biomedcentral.com/submissions 